\title{
Etat de la sensibilité aux antibiotiques des bactéries anaérobies isolées lors d'infections odontogènes. Intérêt de l'association spiramycine-metronidazole
}

\author{
Antibiotic susceptibility patterns of anaerobic bacteria from dental origin. \\ Focus on the spiramycin + metronidazole combination
}

\author{
LUC DUBREUIL ${ }^{1}$, LAURENT CALVET ${ }^{1}$, CHRISTINE ROCQUES ${ }^{2}$ ET PATRICE SEDNAOUI ${ }^{3}$
}

\begin{abstract}
RÉSUMÉ
Le but de cette étude a été de tester l'activité in vitro des antibiotiques suivants : l'amoxicilline seule ou associée à l'acide clavulanique, l'érythromycine, l'azithromycine, la spiramycine, la clindamycine, la pristinamycine, le métronidazole et l'association spiramycine + métronidazole vis-à-vis de 104 souches isolées en 2002 à partir de cellulites d'origine dentaire et dans deux tiers des cas de poches parodontales. Les concentrations minimales inhibitrices (CMI) ont été mesurées par la méthode de dilution en milieu gélosé de Brucella + $5 \%$ de sang. La lecture des $\mathrm{CMl}$ a été réalisée après 48 heures d'incubation en chambre anaérobie. La production de $B$-lactamase a été recherchée à l'aide de disques de nitrocéfine.

La production de B-lactamase chez Prevotella et Porphyromonas est peu fréquente (10 \%). Les taux de résistance les plus élevés sont observés avec l'érythromycine $(53,8 \%)$ dont 20 souches de Fusobacterium et 13 souches de Veillonella, la spiramycine $(41,3 \%)$ dont 9 souches de Fusobacterium et 13 souches de Veillonella. $29 \%$ des souches sont résistantes à la pristinamycine dont 12 souches de Veillonella. 11,5\% des souches à l'azithromycine, 14,4\% au métronidazole dont 12 souches de Propionibacterium acnes et 6,7 \% à la clindamycine. La sensibilité des anaérobies stricts aux macrolides et aux streptogramines est techniquement difficile à établir du fait du $\mathrm{CO} 2$, ce qui rend aléatoire toute comparaison entre ces antibiotiques.

L'association spiramycine + métronidazole est active sur les 47 souches résistantes à la spiramycine, les 15 souches résistantes au métronidazole et sur les 3 souches résistantes à ces deux antibiotiques. Au total $100 \%$ des souches sont sensibles aux associations spiramycine + métronidazole et amoxicilline + acide clavulanique. La grande activité de l'association spiramycine + métronidazole est due à la complémentarité de leurs spectres respectifs et à la synergie totale $(F I C$ index $<0,5)$ ou partielle $(F I C<0,75)$ observée pour respectivement 30 et 43 souches. Cette étude permet d'affirmer le maintien de l'activité des composants de l'association spiramycine + métronidazole sur les anaérobies stricts impliqués dans les infections odontogènes.
\end{abstract} (Med Buccale Chir Buccale 2003; 9: 167-75)

mots clés : Anaérobies, infections dentaires, spiramycine-métronidazole, association d'antibiotiques

\section{SUMMARY}

In this study we compared the in vitro activity of the following agents: amoxicillin alone or combined with clavulanate, erythromycin, azithromycin, spiramycin, clindamycin, pristinamycin, metronidazole and the combination of spiramycin + metronidazole against 104 anaerobic strains isolated from dental abscess or periodontal pockets. Minimal inhibitory concentrations (MICs) were determined by a reference agar dilution method using Brucella blood agar. MICs were determined after 48 hours of incubation in an anaerobic chamber. B-lactamase production was detected using nitrocefin-discs. B-lactamase production among Prevotella and Fusobacterium species was rare

\footnotetext{
${ }^{1}$ Faculté de Pharmacie de Lille

${ }^{2}$ Facultés de Pharmacie de Toulouse

${ }^{3}$ Institut Fournier Paris
}

Demande de tirés à part :

Luc Dubreuil Faculté de Pharmacie de Lille 3 rue Laguesse BP 8359006 Lille cedex 
(10\%). Resistance rates were respectively 53.8\% for erythromycin including 20 strains of Fusobacterium and 13 strains of Veillonella, 41.3\% for spiramycin including 9 strains of Fusobacterium and 13 strains of Veillonella, 11.5 for azithromycin, $14.4 \%$ for metronidazole including 12 Propionibacterium acnes and $6.7 \%$ for clindamycin. Susceptibility of anaerobes to macrolides and streptogramins is difficult to assess due to the CO2 effect, which makes difficult any comparison between antibacterial agents. The spiramycin-metronidazole combination was potent against the 47 erythromycin-resistant strains, the 15 metronidazole resistant strains and the 3 strains resistant to two antibiotics of the combination when used alone. On the whole anaerobes $100 \%$ of the investigated anaerobes were susceptible to the two combinations spiramycin + metronidazole and amoxicillin + clavulanic acid. The good activity of spiramycin plus metronidazole comes from their complementary antibacterial patterns and to the total (FIC index< 0.5) or partial (FIC < 0.75) synergism observed for 30 and 43 strains, respectively. This study confirms the potent activity of the Birodogyl components against anaerobes from dental origin. (Med Buccale Chir Buccale 2003; $9: 167-75)$

key words : Anaerobes, dental infections, spiramycin, metronidazole, antibiotic combination

médecine

buccale

chirurgie

buccale

VOL. $9, \mathrm{~N}^{\circ} 3$ 2003

page 168
La prescription des antibiotiques en odontologie et stomatologie a fait l'objet de recommandations par l'Agence Française de Sécurité Sanitaire des Produits de Santé (AFSSAPS) [1]. Dans les infections de sévérité moyenne, les antibiotiques recommandés en première intention regroupent les aminopénicillines (amoxicilline), les 5-nitro-imidazolés seuls (métronidazole) ou associés aux macrolides et, notamment en cas d'allergie aux B-lactamines, les macrolides, les lincosamides (clindamycine) et les streptogramines (pristinamycine). L'association amoxicilline + acide clavulanique est recommandée en deuxième intention. Les céphalosporines ne sont pas recommandées tandis que les cyclines sont réservées au seul traitement de la parodontite juvénile localisée. L'association spiramycine + métronidazole a été largement utilisée. L'activité de cette association sur les anaérobies de la flore buccale avait été évaluée en 1997 [2] ; il apparaissait indispensable que cette association fasse l'objet d'une nouvelle évaluation. De plus, ces antibiotiques recommandés n'ont jamais été étudiés simultanément. II fallait également apprécier l'activité de la spiramycine et du métronidazole dans le rapport 2/1 qui correspond à la fois au ratio rencontré dans la forme galénique, les taux sanguins et la plupart des taux tissulaires. Le but de cette étude est donc de déterminer les $\mathrm{CMI}$ de 3 macrolides à 14,15 et 16 atomes (respectivement l'érythromycine, l'azithromycine et la spiramycine), d'une lincosamide (clindamycine), d'une streptogramine (pristinamycine), du métronidazole, de l'association spiramycine + métronidazole (2/1) et de I'amoxicilline seule ou associée à l'acide clavulanique sur un collectif de 104 souches anaérobies isolés à partir de cellulites d'origine dentaire et dans deux tiers des cas de poches parodontales. En comparant les CMl de l'association spiramycine + métronidazole aux $\mathrm{CMI}$ de la spiramycine et du métronidazole, il sera possible de voir si l'association est additive, antagoniste ou synergique comme le montrent plusieurs études antérieures ${ }^{[2,3]}$.

\section{MATERIEL ET METHODES}

\section{Souches bactériennes}

Elles ont été isolées à partir de prélèvements pathologiques chez des sujets atteints de parodontites ou de cellulites d'origine dentaire. Cent quatre souches consécutives et non répétitives ont été conservées.

Les souches sont identifiées [4], puis les CMI des antibiotiques sont déterminées par la méthode de référence M11A5 du NCCLS [5].

Afin de s'assurer de la validité des résultats obtenus et de leur reproductibilité, 3 souches de référence préconisées par la norme $M 11 \mathrm{~A} 5 \mathrm{du}$ NCCLS (National Commitee for Clinical and 
Laboratory Standards) sont introduites dans chaque série d'essais : Bacteroides fragilis ATCC 25285, Bacteroides thetaiotaomicron ATCC 29741, Eggerthella lenta ATCC 43055.

\section{Détermination des $\mathrm{CMI}$}

Les CMI sont mesurées par une technique de dilution en milieu gélosé selon la norme M II T du NCCLS et en fonction des recommandations ultérieures de la norme M 11 A5 du NCCLS [5].

\section{- Antibiotiques : préparation de la solu- tion-mère et de ses dilutions}

A partir des poudres titrées fournies par les laboratoires pharmaceutiques et selon leurs propres recommandations, une solution-mère $\left(1280 \mu \mathrm{g} . \mathrm{l}^{-1}\right)$ est préparée. Les dilutions successives au demi seront réalisées en eau distillée stérile à partir de cette solution-mère et selon les directives de Ericsson et Sherris [6].

\section{- Préparation des boîtes de Petri}

Chacune des dilutions d'antibiotique est ensuite incorporée en milieu Brucella au sang préalablement fondu puis ramené à la température de $55^{\circ} \mathrm{C}$, de façon à réaliser, pour chaque antibiotique, une gamme de concentrations. Les gammes de concentration sont les suivantes : amoxicilline et amoxicilline + acide clavulanique (concentration fixe de $2 \mathrm{mg.l}^{\mathrm{I}^{-1}}$ ) de 64 à $0,06 \mathrm{mg} / \mathrm{l}$, érythromycine, spiramycine et pristinamycine de 64 à

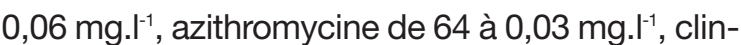

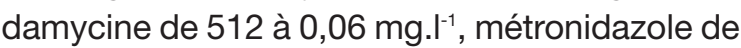
128 à 0,06 mg..$^{-1}$, association spiramycine-metronidazole (dans un rapport 1/2) de 64/32 à 0,125/0,06 mg.l $\mathrm{l}^{-1}$.

Les boîtes ainsi préparées sont séchées $30 \mathrm{~min}$ avant ensemencement. Le stockage de ces boîtes est évité dans la mesure du possible; la durée maximale de stockage des boîtes en anaérobiose ne dépasse pas 24 heures.

\section{- Préparation de l'inoculum}

Une préculture en milieu de Rosenow est diluée en bouillon Brucella de façon à obtenir une opacité voisine de l'échelle 0,5 de Mac Farland. $L^{\prime}$ inoculum contient alors $10^{7}$ à $10^{8}$ UFC. $\mathrm{ml}^{-1}$. Pour certaines souches exigeantes le bouillon
Brucella peut être supplémenté avec $5 \mu \mathrm{g} . \mathrm{I}^{-1} \mathrm{~d}$ 'hémine, $0,1 \mathrm{mg} . \mathrm{l}^{-1}$ de ménadione, $1 \mathrm{mg} . \mathrm{l}^{-1}$ de bicarbonate de sodium et $0,1 \mathrm{ml}$ de sang laqué par tube de $10 \mathrm{ml}$.

\section{- Ensemencement des boîtes de Petri}

A l'aide d'un ensemenceur à têtes multiples de type Steers, on dépose sur les boîtes de Petri 2 à $3 \mu \mathrm{l}$ de l'inoculum préparé comme précédemment et préalablement déposé dans chacune des cupules de l'ensemenceur, de façon à obtenir un inoculum final de $10^{5}$ UFC par spot d'inoculation. Deux séries de deux boîtes sans antibiotique sont également préparées. La première série est ensemencée en tout premier lieu, la deuxième l'est en dernier lorsque toutes les boîtes contenant les antibiotiques sont inoculées. Deux boîtes placées en anaérobiose servent de témoin de croissance; les deux autres incubées en aérobiose permettent de détecter une éventuelle contamination de la souche elle-même ou survenant au cours des manipulations.

\section{- Lecture des résultats}

Elle est réalisée après $48 \mathrm{~h}$ d'incubation en chambre anaérobie pour les souches de Bacteroides du groupe fragilis, Clostridium perfringens, après 72 heures pour les autres espèces bactériennes à croissance plus lente.

La CMI est la plus faible concentration d'antibiotique capable d'inhiber toute croissance visible. La catégorisation des souches est faite selon les recommandations du CA-SFM [7]. Ont été classées résistantes les souches dont les CMI sont supérieures aux concentrations critiques suivantes : érythromycine, azithromycine et spira-

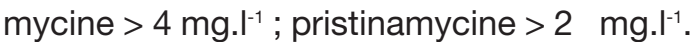

Pour les anaérobies des concentrations critiques spécifiques sont indiquées par le CA-

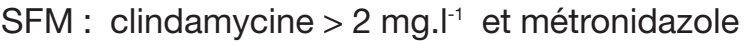
$>16 \mathrm{mg}^{\mathrm{I}^{-1}}$.

En ce qui concerne l'association spiramycine + métronidazole, une souche est résistante lorsque la $\mathrm{CMl}$ est $>8 / 4 \mathrm{mg} / \mathrm{l}$. Une $\mathrm{CMl}$ de l'association à 4/2 correspond à la sensibilité aux deux antibiotiques, une CMI de 8/4 correspond à la résistance au macrolide avec maintien de la sensibilité au métronidazole. \begin{tabular}{l}
$\begin{array}{l}\text { médecine } \\
\text { buccale } \\
\text { chirurgie } \\
\text { buccale }\end{array}$ \\
\hline VOL. $9, N^{\circ} 3$ \\
2003 \\
\hline page 169
\end{tabular} 


\section{Recherche d'une synergie entre la spi- ramycine et le métronidazole}

Lorsque la CMI de la spiramycine ou du métronidazole est basse $\leq 0,06 \mathrm{mg}^{-1} \mathrm{I}^{-1}$ (dernière concentration testée), le FIC index ne peut être calculé. Pour chacune des autres souches, il est possible de déterminer le FIC index (fractionnary inhibitory concentration) ainsi :

$\mathrm{FIC}=\frac{\begin{array}{c}\text { [concentration } \\ \text { de spiramycine } \\ \text { dans l'association] }\end{array}}{\begin{array}{c}\text { CMl de } \\ \text { spiramycine seule }\end{array}}+\frac{\begin{array}{c}\text { [concentration de } \\ \text { métronidazole } \\ \text { dans l'association }]\end{array}}{\begin{array}{c}\text { CMl de } \\ \text { métronidazole seul }\end{array}}$

La synergie totale est définie par une valeur du $\mathrm{FIC}$ index $\leq 0,5$, la synergie partielle $\mathrm{FIC} \leq 0,75$, une addition $\mathrm{FIC} \leq 1$, un antagonisme $\mathrm{FIC}>2$.

médecine

buccale

chirurgie

buccale

VOL. $9, \mathrm{~N}^{\circ} 3$ 2003

page 170

\section{RESULTATS}

Le nombre de souches, l'écart des valeurs de CMI, celles des CMI $50 \%$ et $90 \%$ sont indiqués pour chaque groupe d'espèces dans les tableaux 1 (anaérobies à Gram négatif) et 2 (anaérobies à Gram positif); le nombre de souches résistantes à chacun des antibiotiques étudiés est indiqué dans le tableau 3.

\section{Anaérobies à Gram négatif}

La production de ß-lactamase a été observée pour une souche de Porphyromonas gingivalis et une souche de Prevotella melaninogenica. Toutes les souches de Prevotella et de Porphyromonas étaient sensibles au métronidazole, à la clindamycine et aux deux associations amoxicilline + acide clavulanique et spiramycine + métronidazole. Aucune résistance à la pristinamycine n'était observée chez Prevotella.

Toutes les souches de Fusobacterium étaient sensibles à amoxicilline + acide clavulanique, métronidazole seul ou associé à la spiramycine et clindamycine. Six des 26 Fusobacterium produisaient une pénicillinase qui était inhibée par l'acide clavulanique.

Sur l'ensemble des anaérobies à Gram négatif, aucune résistance n'était observée pour les deux associations métronidazole + spiramycine et amoxicilline + acide clavulanique. Une seule souche de Veillonella était résistante au métronidazole.

\section{Cocci anaérobies à Gram +}

Toutes les souches de Micromonas micros (exPeptostreptococcus micros) étaient sensibles à l'amoxicilline, à l'association spiramycine + métronidazole. La résistance à la clindamycine était observée une fois, celle au métronidazole ou à la clindamycine deux fois, tandis que la résistance croisée aux 3 macrolides était observée trois fois. Les souches d'Anaerococcus prevotii (exPeptostreptococcus prevotii) étaient plus fréquemment résistantes aux macrolides et à la spiramycine. Deux souches étaient résistantes au métronidazole.

\section{Bacilles à Gram +}

Aucune résistance n'était observée avec l'association spiramycine + métronidazole, la clindamycine ou l'amoxicilline.

\section{Synergie de l'association spiramycine- métronidazole}

Lorsque le FIC index était calculable, 30 synergies totales ( $F I C$ index $\leq 0,5$ ) et treize synergies partielles au seuil de 0,512 sont obtenues. L'association est additive ou synergique selon les souches.

\section{DISCUSSION}

\section{Anaérobies à Gram négatif}

Les Bacteroides du groupe fragilis sont rarement isolés de la flore buccale. Une souche de $B$. distasonis et de $B$. uniformis ont été isolées. Ces deux espèces produisent une céphalosporinase chromosomique, inhibable par l'acide clavulanique, qui est responsable de la résistance à I'amoxicilline [8]. Ces espèces sont sensibles au métronidazole et le plus souvent résistantes aux macrolides. La résistance à la clindamycine est de l'ordre de $30 \%$ [9].

La production de $B$-lactamase a été observée pour une souche de Porphyromonas gingivalis et une souche de Prevotella melaninogenica. La Blactamase des Prevotella [10] était responsable de 
Tableau 1 : Activité comparée de 9 antibiotiques sur les anaérobies à Gram négatif Comparative activities of 9 antibiotics against gram-negative anaerobes

\begin{tabular}{|c|c|c|c|c|}
\hline \multirow[b]{2}{*}{ Bacteroides du groupe fragilis a ${ }^{\text {(2) }}$} & \multirow{2}{*}{$\begin{array}{l}\text { Antibiotique } \begin{array}{c}\text { Ecart des CMI } \\
(\mathbf{m g} / \mathbf{L})\end{array} \\
\text { Amoxicilline } \\
\text { Co-amoxiclav } \\
\text { Erythromycine } \\
\text { Azithromycine } \\
\text { Clindamycine } \\
\text { Pristinamycine } \\
\text { Spiramycine } \\
\text { Spiramycine + métronidazole } \\
\text { Métronidazole }\end{array}$} & \multicolumn{2}{|c|}{$\begin{array}{c}\mathrm{CMI}^{*} 50 \% \\
\text { (mg/L) }\end{array}$} & $\begin{array}{l}\mathrm{CMI}^{*} 90 \% \\
\text { (mg/L) }\end{array}$ \\
\hline & & $\begin{array}{l}16-64 \\
0,25-8 \\
16->64 \\
>64 \\
4->256 \\
4-8 \\
32->64 \\
1 / 0,5-2 / 1 \\
0,5-1\end{array}$ & & \\
\hline Porphyromonas spp ${ }^{\circ}$ (6) & $\begin{array}{l}\text { Amoxicilline } \\
\text { Co-amoxiclav } \\
\text { Erythromycine } \\
\text { Azithromycine } \\
\text { Clindamycine } \\
\text { Pristinamycine } \\
\text { Spiramycine } \\
\text { Spiramycine + métronidazole } \\
\text { Métronidazole }\end{array}$ & $\begin{array}{l}\leq 0,06-32 \\
\leq 0,06-0,25 \\
0,25->64 \\
\leq 0,03-8 \\
\leq 0,06-0,5 \\
0,125-8 \\
1-32 \\
\leq 0,125 / 0,06-4 / 2 \\
<0,06-2\end{array}$ & & \\
\hline Prevotella spp ${ }^{d}$ (7) & $\begin{array}{l}\text { Amoxicilline } \\
\text { Co-amoxiclav } \\
\text { Erythromycine } \\
\text { Azithromycine } \\
\text { Clindamycine } \\
\text { Pristinamycine } \\
\text { Spiramycine } \\
\text { Spiramycine + métronidazole } \\
\text { Métronidazole }\end{array}$ & $\begin{array}{l}\leq 0,06->64 \\
\leq 0,06 \\
<0,06-8 \\
<0,03-0,5 \\
\leq 0,6-0,125 \\
\leq 0,06-1 \\
\leq 0,06-8 \\
\leq 0,125 / 0,06- \\
0,25 / 0,125 \\
\leq 0,06-2\end{array}$ & & \\
\hline Fusobacterium spp ${ }^{\circledR}(26)$ & $\begin{array}{l}\text { Amoxicilline } \\
\text { Co-amoxiclav } \\
\text { Erythromycine } \\
\text { Azithromycine } \\
\text { Clindamycine } \\
\text { Pristinamycine } \\
\text { Spiramycine } \\
\text { Spiramycine + métronidazole } \\
\text { Métronidazole }\end{array}$ & $\begin{array}{l}\leq 0,06->64 \\
\leq 0,06 \\
0,06->64 \\
\leq 0,03->64 \\
\leq 0,06-0,125 \\
<0,06-8 \\
\leq 0,06-16 \\
\leq 0,125 / 0,06-2 / 1 \\
\leq 0,06-4\end{array}$ & $\begin{array}{l}\leq 0,06 \\
\leq 0,06 \\
16 \\
0,5 \\
\leq 0,06 \\
1 \\
4 \\
0,25 / 0,125 \\
0,125\end{array}$ & $\begin{array}{l}16 \\
<0,06 \\
32 \\
1 \\
0,125 \\
2 \\
16 \\
0,5 / 0,25 \\
2\end{array}$ \\
\hline Veillonella spp (13) & $\begin{array}{l}\text { Amoxicilline } \\
\text { Co-amoxiclav } \\
\text { Erythromycine } \\
\text { Azithromycine } \\
\text { Clindamycine } \\
\text { Pristinamycine } \\
\text { Spiramycine } \\
\text { Spiramycine + métronidazole } \\
\text { Métronidazole }\end{array}$ & $\begin{array}{l}\leq 0,06-2 \\
\leq 0,06-2 \\
8-64 \\
1-32 \\
\leq 0,06-32 \\
0,5-16 \\
16->64 \\
0,5 / 0,25-8 / 4 \\
\leq 0,06->64\end{array}$ & $\begin{array}{l}\leq 0,06 \\
\leq 0,06 \\
32 \\
2 \\
0,125 \\
8 \\
32 \\
2 / 1 \\
1\end{array}$ & $\begin{array}{l}0,5 \\
0,5 \\
32 \\
4 \\
8 \\
16 \\
64 \\
4 / 2 \\
2\end{array}$ \\
\hline $\begin{array}{l}\text { * valeurs calculées si l'effectif est } \\
\text { a B. distasonis (1 souche), B. unif } \\
\text { " co-amoxiclav = association ame } \\
\text { ' Porphyromonas gingivalis (4 sol } \\
\text { - Prevotella intermedia (3 souche } \\
\text { - Fusobacterium nucleatum (20 s }\end{array}$ & $\begin{array}{l}\text { supérieur à } 10 \text { souches } \\
\text { formis ( } 1 \text { souche) } \\
\text { oxicilline + acide clavulanique } \\
\text { uches), P. assacharolytica ( } 2 \text { souches) } \\
\text { s), P. melaninogenica ( } 3 \text { souches), P. sp } \\
\text { souches), F. varium (6 souches) }\end{array}$ & p (1 souche) & & \\
\hline
\end{tabular}

médecine

buccale chirurgie buccale

VOL. $9, \mathrm{~N}^{\circ} 3$ 2003

page 171 
Tableau 2 : Activité comparée de 9 antibiotiques sur les anaérobies à Gram positif Comparative activities of 9 antibiotics against gram-positive anaerobes

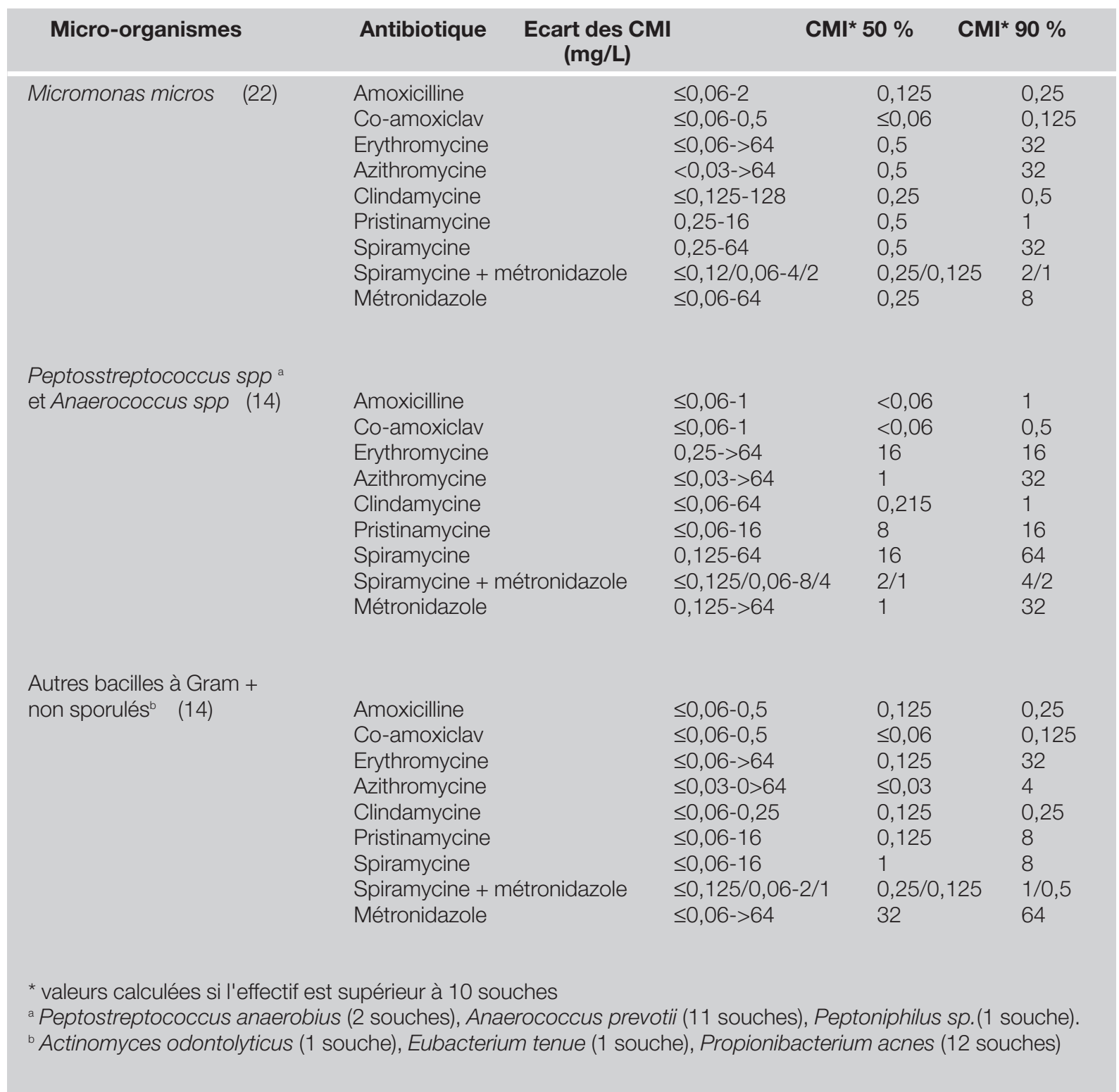

la résistance aux pénicillines et aux céphalosporines [11].

La pristinamycine était active sur 24 des 26 souches étudiées, confirmant d'autres études antérieures [12-13]. Si la résistance naturelle aux macrolides est connue chez Fusobacterium [1214], la sensibilité apparente de l'azithromycine se comprend mal. Si des valeurs de CMI plus basses ont été confirmées par d'autres auteurs [15-16], il est difficile de tirer des conclusions dans la mesure où les macrolides sont hydrolysés en milieu acide et peu stables dans le temps, en solution ou dans les boîtes de Petri. Le dioxyde de carbone nécessaire à la culture des anaérobies acidifie les milieux et provoque une élévation artificielle des $\mathrm{CMI}$ des macrolides les moins stables [17-20].

La quasi-totalité des souches de Veillonella était résistante à l'érythromycine, à la spiramycine et à la pristinamycine. II s'agit d'une résistance naturelle des Veillonella aux macrolides et à la pristinamycine [13,21]. L'azithromycine apparaît plus 
Tableau 3 : Nombre de souches résistantes à chacun des antibiotiques Number of resistant strains to each investigated antibiotic

\begin{tabular}{|c|c|c|c|c|c|c|c|c|c|c|}
\hline Bactéries & $\begin{array}{c}\text { Nombre } \\
\text { de souches }\end{array}$ & AMX & AMC & ERY & AZI & SPI & CLN & PRIST & SPI-MTR & MTR $^{\circ}$ \\
\hline Bacteroides spp. & 2 & 2 & & 2 & 2 & 2 & 2 & 2 & & \\
\hline Poprhyromonas spp. & 6 & 1 & & 4 & 2 & 3 & & 1 & & \\
\hline Prevotella spp. & 7 & 1 & & 1 & 0 & 1 & & & & \\
\hline Fusobacterium nucleatum & 21 & 6 & & 16 & 1 & 7 & & 1 & & \\
\hline F. varium & 5 & & & 4 & & 2 & & 1 & & \\
\hline Veillonella spp. & 13 & & & 13 & 1 & 13 & 2 & 12 & & 1 \\
\hline Ensemble des BGN* & 54 & 10 & 0 & 40 & 6 & 28 & 4 & 17 & 0 & 1 \\
\hline Bacilles à Gram + & 14 & & & 4 & 1 & 3 & & 3 & & 10 \\
\hline Anaerococcus prevotii & 11 & & & 9 & 2 & 9 & 1 & 9 & & 1 \\
\hline Micromonas micros & 22 & & & 3 & 3 & 3 & 2 & 1 & & 2 \\
\hline Autres cocci à Gram + & 3 & & & & & & & & & 1 \\
\hline Ensemble des Gram + & 50 & 0 & 0 & 16 & 6 & 15 & 3 & 13 & 0 & 14 \\
\hline Ensemble des anaérobies & 104 & 10 & 0 & 56 & 12 & 43 & 7 & 30 & 0 & 15 \\
\hline
\end{tabular}

$\mathrm{AMX}=$ amoxicilline, $\mathrm{AMC}=\mathrm{co}$-amoxiclav = amoxicilline + acide clavulanique, $\mathrm{ERY}=$ érythromycine,

$\mathrm{AZI}=$ azithromycine, $\mathrm{SPI}=$ spiramycine, CLN = clindamycine, $\mathrm{PRN}=$ pristinamycine,

SPI-MTR = association spiramycine+ métronidazole, MTR = métronidazole

\begin{tabular}{l}
$\begin{array}{l}\text { médecine } \\
\text { buccale } \\
\text { chirurgie } \\
\text { buccale }\end{array}$ \\
\hline VOL. $9, N^{\circ} 3$ \\
2003 \\
\hline page 173
\end{tabular}

sensible. II est difficile d'expliquer cette différence. Les mécanismes de résistances aux macrolides par inactivation enzymatique font que les enzymes inactivent simultanément les macrolides à 14 et 15 atomes. Lorsqu'il s'agit d'un mécanisme d'efflux rejetant l'érythromycine et la pristinamycine, cela concerne également l'azithromycine. Seule une perméabilité supérieure de l'azithromycine à travers la paroi des anaérobies à Gram négatif pourrait expliquer cette différence. II est beaucoup plus probable que ce soient les conditions méthodologiques qui rendent difficiles l'étude de sensibilité des anaérobies aux macrolides. Sur les bacilles à Gram négatif du contrôle de qualité, des variations importantes sont observées (de 2 à 4 dilutions au demi) des valeurs des $\mathrm{CMI}$ de l'érythromycine et de l'azithromycine, mais pas avec les autres antibiotiques ou les anaérobies à Gram+.

Une des souches de Veillonella était résistante au métronidazole ; cette souche possédait un gène nim E [21].

\section{Bacilles à Gram +}

Les bacilles étaient le plus souvent résistants au métronidazole (résistance naturelle des Actinomyces et propionibactéries). Bien que deux souches étaient également résistantes à la spiramycine, aucune résistance n'était observée avec l'association spiramycine + métronidazole, la clindamycine ou l'amoxicilline.

\section{Ensemble des anaérobies}

Sur l'ensemble des 104 anaérobies isolées d'infections odontogènes, la résistance à l'érythromycine était observée 56 fois. II s'agit d'une résistance naturelle pour 20 souches de Fusobacterium et 13 souches de Veillonella. II y a donc 23 cas de résistance acquise. La situation était comparable pour la spiramycine : 43 souches résistantes dont 9 Fusobacterium et 13 Veillonella. La pristinamycine était plus active : 30 souches résistantes dont 12 souches de Veillonella. Parmi les 15 souches résistantes au métronidazole, on trouve 12 souches de $P$. acnes (résistance natu- 
relle). La production de $ß$-lactamase responsable de la résistance à l'amoxicilline est observée pour 10 bacilles à Gram négatif sur 54 souches soit $18,5 \%$. L'association spiramycine + métronidazole était active sur tous les anaérobies de cette étude y compris sur les 15 souches résistantes au métronidazole, les 43 souches résistantes à la spiramycine. Les deux antibiotiques ont donc une complémentarité de spectre antibactérien. II est à noter qu'une souche de Veillonella et d'Anaerococcus prevotii et deux souches de $P$. acnes étaient résistantes aux deux antibiotiques. Ces quatre souches sont sensibles à leur association. De même, aucune résistance n'est observée avec l'association amoxicilline + acide clavulanique.

médecine buccale chirurgie buccale

VOL. $9, \mathrm{~N}^{\circ} 3$ 2003

page 174

\section{RÉFÉRENCES}

1 - Agence l'agence Française de Sécurité Sanitaire des Produits de santé (AFSSAPS). Prescription des antibiotiques en odontologie et stomatologie. Recommandations et argumentaire. AFSSAPS, Juillet 2001.

2 - ROCHE Y, YOSHIMORI RN. In vitro activity of spiramycin and metronidazole alone or in combination against isolates from oodontogenic abscesses. J Antimicrob Chemother 1997; 40: 353-7.

3 - BROOK I. Synergy between spiramycin and metronidazole in the treatment of polymicrobial infections. $J$ Antimicrob Chemother 1998 ; 22 suppl B,78-85.

4 - Summanen P., Baron E.J., Citron D.M., Strong C.A., WEXLER H.M. AND FINEGOLD S.M. (1993). Wadsworth anaerobic bacteriology manual, 5th ed. Star Publishing Co., Belmont, California.

5 - National Committee for Clinical Laboratory Standards. (2001). Methods for antimicrobial susceptibility testing of anaerobic bacteria; Approved standard - fifth edition. NCCLS document M11-A5 [ISBN 1-56238-4295]. NCCLS, 940 West Valley Road, Suite 1400, Wayne, Pennsylvania 19087-1898, USA.

6 - ERICSON HM, SHERRIS JC. Antibiotic sensitivity testing report of an international collaborative study. Acta Pathol Microbiol Scand 1971; sect B, suppl 27.

7 - Comité de l'Antibiogramme de la Société Française de Microbiologie Report 2003. Int. J. Antimicrob. Agents. 2003; 21: 364-91

8 - Rogers MB, PARKer AC, SMith CJ. Cloning and characterization of the endogenous cephalosporinase gene, cepA, from Bacteroides fragilis reveals a new subgroup
Cette étude confirme l'existence de souches productrices de B-lactamase inactivant l'amoxicilline chez les anaérobies à Gram négatif. On observe un taux de résistance non négligeable aux macrolides essentiellement en raison de la résistance naturelle de certaines espèces (Veillonella et Fusobacterium). L'activité de la clindamycine est bonne (taux de résistance 6,7 \%). Aucune souche n'est résistante aux associations amoxicilline + acide clavulanique ou spiramycine + métronidazole. Cette dernière associe deux antibiotiques complémentaires qui agissent souvent en synergie. Alors que la résistance aux antibiotiques augmente chez les anaérobies, l'activité de l'association spiramycine + métronidazole s'est maintenue sur les anaérobies isolés dans les infections odontogènes.

of Ambler class A B lactamases. Antimicrob. Agents Chemother. 1993; 37: 2391-400.

9 - Behra-Miellet J, Calvet L, Mory F, Muller C, Chomarat M, Bézian MC, Bland S, Juvenin ME, Fosse T, GoldsteIn F, JAULhaC B, DuBREUIL L. Antibiotic resistance among anaerobic Gram negative bacilli : Lessons from a french multicentric survey. Anaerobe 2003; 9 : 105-11.

10 - Valle G, Quiros LM, Andres MT, Fierro JF. A B-lactamase belonging to group $2^{e}$ from oral clinical isolates of Prevotella intermedia. FEMS Microbiol Lett 1998; 151 : 191-4.

11 - Dubreuil L, Behra-Miellet J, Vouillot C, Bland S, Sedallian A, Mory F. B-lactamase production in Prevotella and in vitro susceptibilities to selected betalactam antibiotics. Int J Antimicrob Agents 2003; 21: 267-73.

12 - WeBer P, Dubois S, Boussougant Y. In-vitro activity of pristinamycin and its components against gram-negative anaerobic bacilli and Gardnerella vaginalis. J Antimicrob Chemother 1989; 23: 825-30.

13 - Dubreuil L, Houcke I, Singer E. Activité in vitro de 10 antibiotiques dont la pristinamycine et ses deux composants (RP 12536 et RP 27404) sur les anaérobies stricts. Pathol Biol 1998; 46 : 147-52.

14 - Williams JD, MASKELl JP, Shain H, Chrysos G, Sefton AM, Fraser HY, HARDIE JM. Comparative in-vitro activity of azithromycin, macrolides (erythromycin, clarithromycin and spiramycin) and streptogramin RP 59500 against oral organisms. J Antimicrob Chemother 1992; 32: 27-37. 
15 - Goldstein EJC, Citron DM, Gerardo HS, Hudspeth M, MERRIAM VC. Activities of HMR 3004 (RU 64004) and HMR 3647 (RU 66647) compared to those of erythromycin, azithromycin, clarithromycin, roxithromycin, and eight other antimicrobial agents against unusual aerobics and anaerobics human and animal bite pathogens isolated from skin and soft tissue infections in humans. Antimicrob Agents Chemother 1998; 42: 1127-32

16 - Goldstein EJC, Citron DM, Merriam VC. Linezolid activity compared to those of selected macrolides and other agents against aerobic and anaerobic pathogens isolated from soft tissue bite infections in humans. Antimicrob Agents Chemother 1999; 43: 1469-74.

17 - Felmingham D, Robbins MJ, Marais R, Ridgway GL, GRUNEBERG RN. The effect of carbon dioxide on the in vitro activity of erythromycin and $\mathrm{RU}$-28965 against anaerobic bacteria. Drugs Exp Clin Res 198 ; 13: 195-9.

18 - EdNIE LM, Jacobs MR, AppelBaUm PC. Anti-anaerobic activity of erythromycin, azithromycin and clarithromycin: effect of $\mathrm{pH}$ adjustment of media to compensate for $\mathrm{pH}$ shift caused by incubation in CO2. J Antimicrob Chemother 1998; 41: 387-9.
19 - Johnson MM, HILL SL, PIDdock LJ. Effect of carbon dioxide on testing susceptibilities of respiratory tract pathogens to macrolide azalide antimicrobial agents. Antimicrob Agents Chemother 1999; 43: 1862-5.

20 - BeMER-MECLCHIOR P, JuVIN ME, TASsin S, BRYSKIER A, SCHITO GC, DRUGEON HB. In vitro activity of a new ketolides telithromycin compared with those of macrolides against Streptococcus pyogenes: influences of resistance mechanism and methodological factors. Antimicrob Agents Chemother 2000; 44: 2999-3002.

21 - Goldstein EJC, Citron DM, Merriam CV,Tyrrell K, WARREN Y. Activities of Gemifloxacin (SB 265805 and LB 20304) compared to those of other antimicrobial agents against unusual anaerobes. Antimicrob Agents Chemother 1999; 43: 2726-30.

22 - Marchandin H, Jumas-BILAK E, Teyssier C, JeanPierre H, Mory F, Siméon de Buochberg M, Carrière C, DUBREUIL L. Detection of nim genes in clinical isolates of Veillonella sp. and Bacteroides fragilis, in France. Anaerobe Olympiad 2022. Anaerobe Society of the Americas, Park City, UY, USA, Abstract VO-1. \begin{tabular}{l}
$\begin{array}{l}\text { médecine } \\
\text { buccale } \\
\text { chirurgie } \\
\text { buccale }\end{array}$ \\
\hline VOL. $9, N^{\circ} 3$ \\
2003
\end{tabular}

page 175 